\title{
Review
}

\section{Towards Personalized Rehabilitation for Gait Impairments in Parkinson's Disease}

\author{
Jorik Nonnekes ${ }^{\mathrm{a}, \mathrm{b}, *}$ and Alice Nieuwboer ${ }^{\mathrm{c}}$ \\ ${ }^{a}$ Radboud University Medical Centre, Donders Institute for Brain, Cognition and Behaviour, \\ Department of Rehabilitation, Nijmegen, The Netherlands \\ ${ }^{\mathrm{b}}$ Department of Rehabilitation, Sint Maartenskliniek, Nijmegen, The Netherlands \\ ${ }^{\mathrm{c}}$ Department of Rehabilitation Sciences, Katholieke Universiteit Leuven, Tervuursevest, Belgium
}

Accepted 24 October 2018

\begin{abstract}
Non-pharmacological interventions are essential in the management of gait impairments in Parkinson's disease. The evidence for these interventions is growing rapidly. However, studies evaluating these interventions do generally evaluate a one-size-fits-all concept, and do usually not distinguish between subgroups, treatment dose and delivery mode. For two main reasons, this approach will not reach the full potential of gait rehabilitation. First, non-pharmacological interventions (e.g., external cueing) can improve gait in certain patients, but have no effect or sometimes even exacerbate gait deficits in others. Second, the success and benefit of gait rehabilitation relies on therapy adherence and training intensity achieved, and multitarget therapy not tailored to the individual runs the risk of hitting nothing. Hence, to apply non-pharmacological interventions in an individualized and evidence-based manner, clinicians and therapists need to know which patient characteristics predict the efficacy of various training modes and what type of training delivery works best. Current evidence is not sufficient to develop such personalized rehabilitation programs. In this viewpoint, however, we describe how tailored use of gait rehabilitation can be reached within a 20 -year time frame.
\end{abstract}

Keywords: Gait, freezing of gait, rehabilitation, Parkinson's disease, personalized medicine, physiotherapy, balance, falls

\section{INTRODUCTION}

Gait impairments in Parkinson's disease (PD) are common and disabling, and range from a reduced step length and height to episodes of freezing of gait $[1,2]$. These gait impairments are disabling as they result in a reduced mobility, falls and fall-related injuries [3, 4]. Optimal management is complex and challenging, involving a combination of pharmacological, non-pharmacological and sometimes surgical interventions [5]. Non-pharmacological interventions are essential, as gait impairments only partially improve

\footnotetext{
*Correspondence to: Jorik Nonnekes, MD, PhD, Radboud University Medical Centre, PO Box 9101, 6500 HB Nijmegen, The Netherlands. E-mail: jorik.nonnekes@radboudumc.nl.
}

in response to (dopaminergic) medication and deep brain stimulation. These interventions are ideally delivered using a multidisciplinary approach (see Panel 1) [6].

In recent years, evidence on rehabilitation for gait impairments in PD has been growing, particularly for physiotherapy [13]. However, studies in this domain generally do not distinguish between subgroups, treatment dose and delivery mode and usually support a one-size-fits-all concept. To reach its full potential, non-pharmacological interventions need to be tailored for two main reasons. First, training interventions can improve gait in certain patients, but have no effect or sometimes even exacerbate gait deficits in others. This has been well documented for external cueing strategies, as cueing can create attentional 
Panel 1

Non-Pharmacological Interventions for Gait Impairments in PD

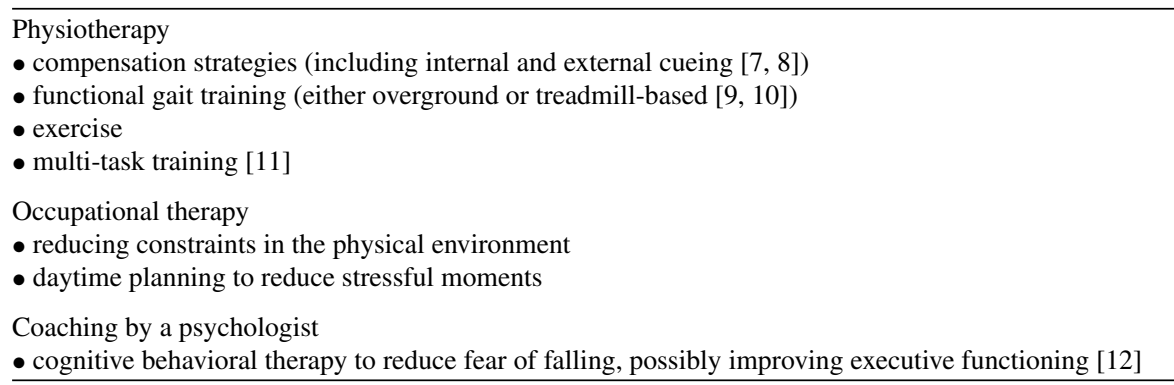

overload [14]. According to our experience, differential treatment effects are also seen in response to other non-pharmacological interventions, such as cognitive behavioral therapy. Second, the success and benefit of non-pharmacological interventions relies on therapy adherence and training intensity achieved. For example, a recent study demonstrated that treadmill training at high intensity alone was able to impact on disease progression and not at moderate intensity [15]. As patients with PD generally have a limited amount of energy, multi-target therapy not tailored to the individual, runs the risk of hitting nothing. Hence, the complexity of designing and applying non-pharmacological interventions is in our view underestimated.

\section{ARE WE READY TO APPLY PERSONALIZED REHABILITATION?}

To apply non-pharmacological interventions in an individualized and evidence-based manner, clinicians and therapists need to know which patient characteristics predict the efficacy of various training modes and what type of training delivery works best. The available evidence is unfortunately sparse in this area and restricted to a limited number of studies. Due to this knowledge-gap, current guidelines do not give recommendations on individualized gait rehabilitation. A recent study looked at the mode of delivery of physiotherapy comparing unsupervised home exercise, versus group training (one-size-fitsall) and one-on-one supervised sessions while the content of training was kept the same [16]. It showed that group training is beneficial for patients needing gait training only, whereas individual exercise is most effective for patients with complex disease and those who suffer from co-morbidity. Unsupervised home exercise was not beneficial. Indeed, patients with apathy and depression report more difficulty to exercise independently at home [17].

When looking at the diversity of the contents of training-based therapy for gait impairments, freezers perform better with closed-loop cueing strategies (cues that are fixed during one session) compared to open-loop strategies (cues that adapt to the gait pattern during one session) [18]. No differences were found for non-freezers. Moreover, optimal cueing frequencies for freezers are somewhat lower than the baseline step frequency, whereas non-freezers perform optimally with a cueing frequency $10 \%$ above their baseline stepping frequency [11]. When employing strategy-training, therapy is most effective in patients with more disease severity (possibly more in need for compensation) and less cognitive decline [19]. Similarly and with respect to dual-task training, secondary analyses of the DUALITY study revealed that patients with slow dual-task gait velocity and a high level of cognitive functioning at baseline are most likely to benefit from dual-task training [20]. Moreover, in the DUALITY study, freezers showed less retention of dual-task training compared to nonfreezers [11], a finding which was also shown after writing training [21]. With respect to exercise for fall prevention, it has been demonstrated that a minimally supervised exercise program results in fewer falls in the lower disease severity subgroup (based on UPDRS motor score at baseline, dichotomized at the median), whereas patients in the higher disease subgroup tended to fall more often. It is obvious that the evidence summarized in this paragraph is far from sufficient to develop personalized rehabilitation programs. We envision, however, that within a 20 -years time frame, evidence-based personalized rehabilitation for gait impairments is common practice. In the remainder of this commentary, we will describe how personalized rehabilitation may look like, and how we can reach it within the next decades. 


\section{HOW MAY PERSONALIZED REHABILITATION BE DELIVERED?}

We foresee that within a personalized rehabilitation approach, every patient with PD is regularly screened for disabling gait impairments using a comprehensive test battery involving both history taking and physical examination. Five domains will need to be evaluated and mapped (see Fig. 1). Factors in each of these domains influence the severity or consequences of gait impairments. The first domain involves motor-symptoms. In its most simple form, this involves freezing of gait, balance impairments and disease severity. The second domain involves non-motor symptoms, which includes cognitive dysfunction, anxiety and mood disturbances. Obviously, relevant co-morbidity (domain 3 ) also impacts on gait impairments in PD. The presence of orthopedic problems such as foot deformities or scoliosis, the presence of muscle weakness and a measure of cardiopulmonary condition needs to be evaluated. The fourth domain evaluates environmental factors, such as constraints in the physical environment and the use of walking aids. When applying a tailored rehabilitation approach, personal, cultural and environmental elements should be taking into account as well (domain 5; see Fig. 1). This involves the personal preferences and aims of the individual patient. An example of a common personal preference is that compensation strategies should not be visible to others (which makes visual cueing strategies to be a less suitable intervention). Other examples of personal aims are the ambition to increase gait speed; the desire to reduce the number of freezing episodes in specific circumstances (shopping, gardening) or the aim to prevent falls when walking outdoors. Other factors involve the ability to travel and the presence or absence of a caregiver. Moreover, knowing that gait impairments can be affected by constraints in the physical environment, living circumstances (e.g., furnishing requiring narrow turns) should be evaluated. A final element is cultural background, as this may also impact the effect of non-pharmacological interventions [22].

After collection of the outcomes of the comprehensive test battery, a treatment algorithm will

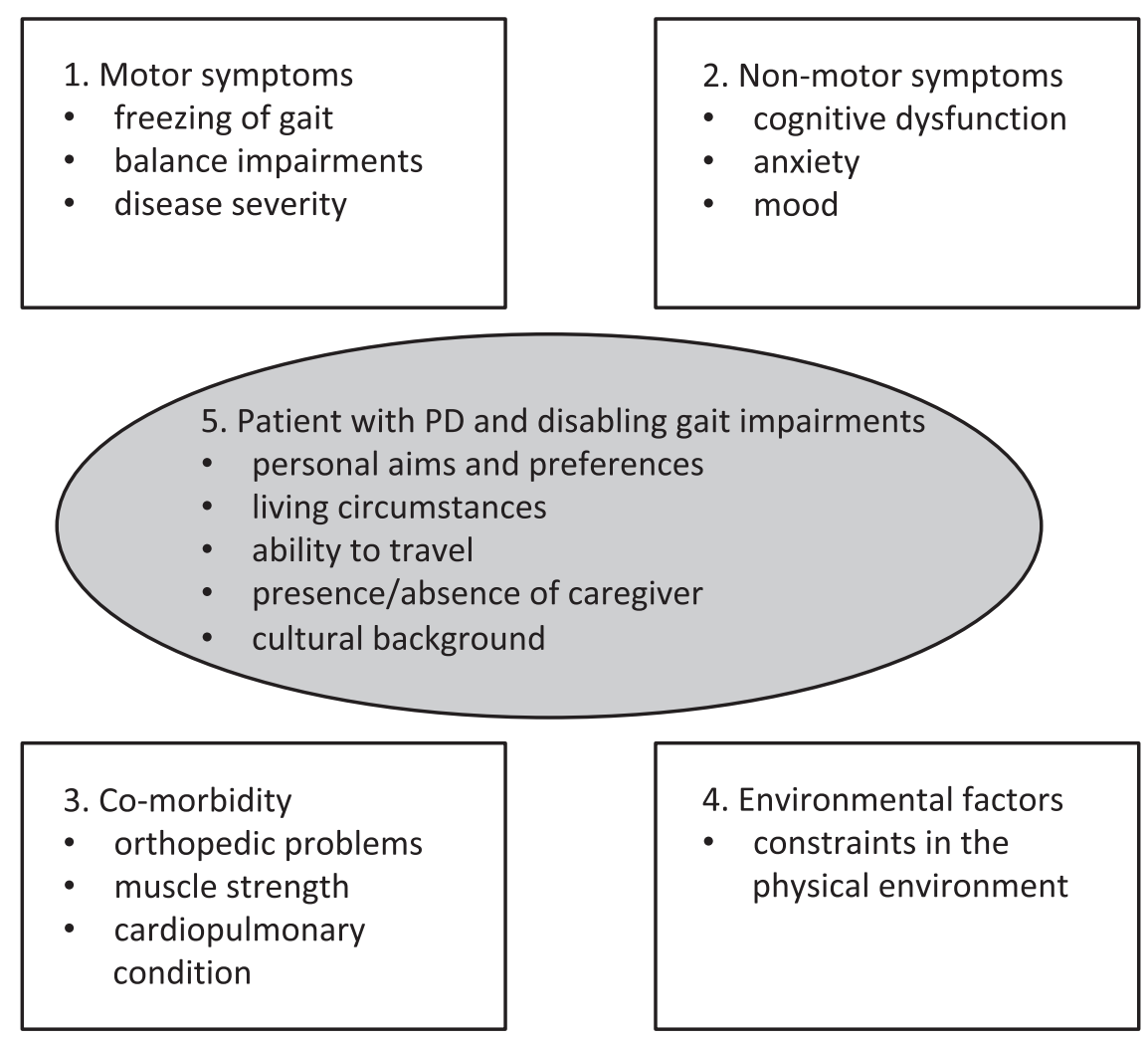

Fig. 1. Potential factors influencing effectiveness of non-pharmacological intervention of gait impairments. Various domains that impact on gait deficits in Parkinson's disease. 
subsequently assist to determine the most effective combination of non-pharmacological interventions (see Panel 2 for two examples). In this treatment algorithm, assessment, preferably based on easy to use cut-off criteria of behavioral tests, determines priority when there are impairments in multiple domains. Moreover, combinations of symptoms in each of the domains can give direction for the type of nonpharmacological interventions to be prescribed. For example, patients with a combination of anxiety and difficulties multi-tasking may benefit from VR-based treadmill training involving dual tasks, as this enables a safe environment with a controlled build-up of tasks (this hypothesis needs to be confirmed by future studies). Patients with freezing of gait and mild executive dysfunction may hypothetically benefit from external cueing strategies, as such strategies may help to assist in filtering information and prioritizing a stimulus, especially during response selection under conflict. However, severe cognitive problems may preclude such intervention.

Current treatment algorithms are usually depicted as a decision tree, such as those recently published in medical journals [5, 23]. It is the question whether such a (printed) decision tree is also feasible for the algorithm described above, as the test battery yields multiple behavioral outcomes that may be difficult to catch and integrate when applied to a rehabilitation setting. In addition, it may be problematic to place the outcomes in a hierarchical order, because it is the combination of outcomes that will determine the type of intervention and the type of training, which may involve many parameters. We envision that a smartphone-based treatment algorithm may be more feasible in the future, when more evidence is available. In this application, which might be an integrated part of future guidelines, the outcomes of the test battery can be entered, including information directly derived from wearable movement sensors. Based on such datasets, suggestions for the most suitable interventions are generated. Importantly, these suggestions do not replace clinical reasoning, but can be used to support it. An advantage is that smartphone-based applications can embed digitized information as well as easily be updated with evidence from the latest studies. Moreover, it can also take the treatment facilities that are available into account (e.g., not every center will have VR-based treadmill training facilities).

\section{HOW TO REACH EVIDENCE-BASED PERSONALIZED REHABILIATION}

As outlined above, only a limited number of studies have reported which patient subgroup benefited most from the studied non-pharmacological intervention. Instead, the majority of studies only report outcomes at group level, whereas a large variation in effect size is usually present. Therefore, the treatment algorithm proposed above cannot yet be designed. Researchers should therefore investigate and report whether outcomes differed between patient subgroups. To this aim, we encourage them to map the motor and nonmotor symptoms described in (Fig. 1). For all of these symptoms, there is no consensus on the most optimal outcome measure. In an attempt to improve uniformity and comparability, we here suggest to (at least)

Panel 2

Examples of Tailored Non-Pharmacological Interventions

Patient A

Patient A is 60-year old woman with a 7-year history of Parkinson's disease. Since one year, she experiences disabling episodes of freezing of gait, which particularly occur during turning in narrow spaces. There is a strong fear to freeze when being in crowded places and when under time pressure. Her right leg is $4 \mathrm{~cm}$ shorter compared to the left leg, which induces asymmetry when walking and turning. Her aim is to reduce the number of episodes of freezing, and she prefers compensation strategies that are not visible to others. Using the treatment algorithm, a tailored rehabilitation program is developed. This involves training of goal-directed weight shifts when turning. Moreover, external rhythmic vibrational cueing is applied to minimize episodes of FOG. In addition, she receives six sessions of cognitive behavioral therapy to reduce her fear of freezing, and two sessions of occupational therapy to reduce stressful moments using daytime planning. Symmetry of walking is restored by a shoe adaptation that compensates for the difference in leg length.

\section{Patient $B$}

This is a 72-year old man with a 4-year history of Parkinson's disease. His gait impairments are continuous in nature; there is no freezing of gait. There is moderate cognitive dysfunction and he has difficulties to perform a dual task when walking (e.g., walking when talking). This has resulted in a fall in the preceding month. There is a reduced cardiopulmonary condition. His aim is to increase his gait speed. He has limited amount of energy, so training efforts should be carefully managed and targets prioritized. Using the treatment algorithm, a tailored program is developed. This involves VR-based exercise training to improve his cardiopulmonary condition, train his cognitive capabilities while at the same time improving multi-tasking when walking. 
use the N-FGOQ to map gait freezing, the MiniBest for balance performance, the MDS-UPDRS for disease severity, the MOCA for cognitive function and the HADS-A and HADS-D for anxiety and mood. However, for the future, we foresee that wearable sensor-based technology, both applied during standard testing and free-living circumstances [24, 25], can provide more precise input into treatment decision-making and refine algorithm development.

We are aware that studies on non-pharmacological interventions are often relatively small, and not powered to perform subgroup analyses. The solution is twofold. First, larger (and powered) studies are recommended, as these are likely to be more informative than small studies that include only 10-15 patients. Second, we encourage groups to share their data-including the collected outcome measures for motor and non-motor symptoms-as the combined analysis of studies may enable to identify relevant subgroups.

In addition, to reach evidence-based personalized rehabilitation, RCTs are needed which compare the effectiveness of one-size-fits all rehabilitation programs with tailored personalized rehabilitation programs involving treatment algorithms described above. These RCTs should not only evaluate effectiveness on the short-term (e.g., effects directly after the intervention), but also on the long-term (6-12 months after the intervention). Moreover, as female patients appear to be underrepresented in PD-trials [26], efforts should be taken to included women into RCTs. Finally, such RCTs need to determine the most optimal intensity, setting (e.g., home-based, inpatient or outpatient rehabilitation setting), frequency and duration of the non-pharmacological interventions.

\section{CONCLUSION}

We have briefly described how tailored use of nonpharmacological interventions can be reached within a 20-year time frame. Importantly, the concept of personalized rehabilitation fits well in major international developments in healthcare [27]. Here, we have focused on the treatment for gait impairments in PD, but an individualized approach, based on a set of behavioral markers, is reasonably also applicable to therapy for other motor and non-motor features in PD. In the next few decades, further technological advance in wearable aids will both refine assessment and clinical reasoning and can be anticipated to support such personalized approaches.

\section{CONFLICT OF INTEREST}

The authors have no conflict of interest to report.

\section{REFERENCES}

[1] Nutt JG, Bloem BR, Giladi N, Hallett M, Horak FB, Nieuwboer A (2011) Freezing of gait: Moving forward on a mysterious clinical phenomenon. Lancet Neurol 10, 734744.

[2] Nonnekes J, Goselink RJM, Ruzicka E, Fasano A, Nutt JG, Bloem BR (2018) Neurological disorders of gait, balance and posture: A sign-based approach. Nat Rev Neurol 14, 183-189.

[3] Bloem BR, Hausdorff JM, Visser JE, Giladi N (2004) Falls and freezing of gait in Parkinson's disease: A review of two interconnected, episodic phenomena. Mov Disord 19, 871-884.

[4] Canning CG, Paul SS, Nieuwboer A (2014) Prevention of falls in Parkinson's disease: A review of fall risk factors and the role of physical interventions. Neurodegener Dis Manag 4, 203-221.

[5] Nonnekes J, Snijders AH, Nutt JG, Deuschl G, Giladi N, Bloem BR (2015) Freezing of gait: A practical approach to management. Lancet Neurol 14, 768-778.

[6] van der Marck MA, Klok MP, Okun MS, Giladi N, Munneke M, Bloem BR, Force NPFFT (2014) Consensus-based clinical practice recommendations for the examination and management of falls in patients with Parkinson's disease. Parkinsonism Relat Disord 20, 360-369.

[7] Ginis P, Nackaerts E, Nieuwboer A, Heremans E (2017) Cueing for people with Parkinson's disease with freezing of gait: A narrative review of the state-of-the-art and novel perspectives. Ann Phys Rehabil Med. doi: 10.1016/j.rehab.2017.08.002

[8] Rahman S, Griffin HJ, Quinn NP, Jahanshahi M (2008) The factors that induce or overcome freezing of gait in Parkinson's disease. Behav Neurol 19, 127-136.

[9] Studer V, Maestri R, Clerici I, Spina L, Zivi I, Ferrazzoli D, Frazzitta G (2017) Treadmill training with cues and feedback improves gait in people with more advanced Parkinson's disease. J Parkinsons Dis 7, 729-739.

[10] Mehrholz J, Kugler J, Storch A, Pohl M, Hirsch K, Elsner B (2015) Treadmill training for patients with Parkinson's disease. Cochrane Database Syst Rev, CD007830.

[11] Strouwen C, Molenaar EALM, Munks L, Keus SHJ, Zijlmans JCM, Vandenberghe W, Bloem BR, Nieuwboer A (2017) Training dual tasks together or apart in Parkinson's disease: Results from the DUALITY Trial. Mov Disord 32, 1201-1210.

[12] Walton CC, Mowszowski L, Gilat M, Hall JM, O'Callaghan C, Muller AJ, Georgiades M, Szeto JYY, Ehgoetz Martens KA, Shine JM, Naismith SL, Lewis SJG (2018) Cognitive training for freezing of gait in Parkinson's disease: A randomized controlled trial. NPJ Parkinsons Dis 4, 15.

[13] Bloem BR, de Vries NM, Ebersbach G (2015) Nonpharmacological treatments for patients with Parkinson's disease. Mov Disord 30, 1504-1520. 
[14] Nombela C, Hughes LE, Owen AM, Grahn JA (2013) Into the groove: Can rhythm influence Parkinson's disease? Neurosci Biobehav Rev 37, 2564-2570.

[15] Schenkman M, Moore CG, Kohrt WM, Hall DA, Delitto A, Comella CL, Josbeno DA, Christiansen CL, Berman BD, Kluger BM, Melanson EL, Jain S, Robichaud JA, Poon C, Corcos DM (2018) Effect of high-intensity treadmill exercise on motor symptoms in patients with de novo Parkinson disease: A phase 2 randomized clinical trial. JAMA Neurol 75, 219-226.

[16] King LA, Wilhelm J, Chen Y, Blehm R, Nutt J, Chen Z, Serdar A, Horak FB (2015) Effects of group, individual, and home exercise in persons with Parkinson disease: A randomized clinical trial. J Neurol Phys Ther 39, 204-212.

[17] Leavy B, Roaldsen KS, Nylund K, Hagstromer M, Franzen E (2017) "Pushing the Limits": Rethinking motor and cognitive resources after a highly challenging balance training program for Parkinson disease. Phys Ther 97, 81-89.

[18] Ginis P, Heremans E, Ferrari A, Bekkers EMJ, Canning CG, Nieuwboer A (2017) External input for gait in people with Parkinson's disease with and without freezing of gait: One size does not fit all. J Neurol 264, 1488-1496.

[19] Nieuwboer A, De Weerdt W, Dom R, Bogaerts K (2002) Prediction of outcome of physiotherapy in advanced Parkinson's disease. Clin Rehab 16, 886-893.

[20] Strouwen C ME, Münks L, Broeder S, Ginis P, Bloem BR, Nieuwboer A, Heremans E (2018) Determinants of dualtask training effect size in Parkinson's disease: Who will benefit most? J Neurol Phys Ther, in press.
[21] Heremans E, Nackaerts E, Vervoort G, Broeder S, Swinnen SP, Nieuwboer A (2016) Impaired retention of motor learning of writing skills in patients with Parkinson's disease with freezing of gait. PLoS One 11, e0148933.

[22] Khalil H, Bajwa JA (2015) Barriers and facilitators in physical rehabilitation for Parkinson's disease in the Arabian world. Mov Disord Clin Pract 2, 227-229.

[23] Nonnekes J, Benda N, van Duijnhoven H, Lem F, Keijsers N, Louwerens JWK, Pieterse A, Renzenbrink B, Weerdesteyn V, Buurke J, Geurts ACH (2018) Management of gait impairments in chronic unilateral upper motor neuron lesions: A review. JAMA Neurol 75, 751-758.

[24] Del Din S, Godfrey A, Mazza C, Lord S, Rochester L (2016) Free-living monitoring of Parkinson's disease: Lessons from the field. Mov Disord 31, 1293-1313.

[25] Horak FB, Mancini M (2013) Objective biomarkers of balance and gait for Parkinson's disease using body-worn sensors. Mov Disord 28, 1544-1551.

[26] Tosserams A, Araujo R, Pringsheim T, Post B, Darweesh SKL, IntHout J, Bloem BR (2018) Underrepresentation of women in Parkinson's disease trials. Mov Disord. doi: 10.1002/mds. 27505

[27] Hamburg MA, Collins FS (2010) The path to personalized medicine. $N$ Engl J Med 363, 301-304. 